\title{
High quality factors and room-temperature lasing in a modified single-defect photonic crystal cavity
}

\author{
Marko Lončar, * Michael Hochberg, and Axel Scherer \\ Departments of Electrical Engineering and Applied Physics, California Institute of Technology, MS 136-93, Pasadena, California 91125
}

\section{Yueming Qiu}

In Situ Technology and Experiments Systems Section, Jet Propulsion Laboratory, California Institute of Technology, MS 302-306, Pasadena, California 91109

Received October 7, 2003

\begin{abstract}
We propose and analyze a new photonic crystal cavity design that supports a dipole mode with a quality factor greater than 20,000. Such a high quality factor is obtained by precise tuning of the cavity length with minimal disruption of the surrounding photonic crystal. A fabrication procedure based on dry etching of InGaAsP material in $\mathrm{HI} / \mathrm{H}_{2} / \mathrm{Ar}$ is used to demonstrate photonic crystal lasers with smooth and straight sidewalls. These room-temperature lasers concentrate optical energy in air and are suitable for use as tunable lasers and chemical sensors. (C) 2004 Optical Society of America

OCIS codes: $140.4780,140.5960$.
\end{abstract}

High-quality-factor $(Q)$ cavities based on planar photonic crystals (PPCs) recently attracted significant research attention because of their ability to store light efficiently, both in space and in time. Several designs with $Q>10,000$ were recently reported. ${ }^{1-5}$ Owing to the high $Q$ and the small mode volumes of PPC cavities $\left(V_{\text {mode }}\right)$, low-threshold room-temperature lasing was also observed in these cavities when they were optically pumped..$^{3-6}$ In the high- $Q$ cavity designs proposed by our group a line defect [fractional edge dislocation (FED)] was introduced into a photonic crystal cavity to enhance the cavity $Q .{ }^{1}$ This design resulted in experimental measurement of high quality factors $(Q \approx 2800){ }^{7}$ low-threshold lasers ${ }^{8}$ $\left(P_{\text {th }} \approx 200 \mu \mathrm{W}\right)$, and chemical sensors. ${ }^{9}$ One disadvantage of this cavity design, however, lies in the broken symmetry of the triangular lattice as a result of the elongation of the central row of holes. The introduced dislocation is expected to pose significant problems for the achievement of photonic circuits or quantum networks, in which efficient integration of several cavities and waveguides is of interest. In this Letter we propose a new high- $Q$ cavity design (Fig. 1) that does not disrupt the symmetry of the triangular lattice.

The PPC cavities that we consider in this work are defined in an InGaAsP membrane suspended in air. The geometry of the triangular photonic crystal lattice surrounding our cavities is $r / a=0.3$ and $d / a=0.75$, where $r$ is the radius of photonic crystal holes, $d$ is the thickness of the membrane, and $a$ is the periodicity of the lattice. A smaller hole with radius $r_{\text {def }} / a=0.15$ is introduced to form an optical cavity. Such a single-defect PPC cavity is known to support two doubly degenerate dipole modes ${ }^{10}$ with modest quality factors. The $x$ dipole mode is usually defined as one where the electric field is polarized along the $x$ axis, whereas the electric field is polarized along the $y$ axis for the $y$ dipole mode. By modifying the lattice, it is possible to lift the degeneracy between these two modes ${ }^{1,8,10}$ and to increase the $Q$ factor of one of these modes at the expense of the other one. In our previous publication ${ }^{1}$ we showed that it is possible to increase $Q$ by tuning the cavity length by elongating one row of holes, thus introducing the dislocation in the lattice. An intuitive way to understand the origin of the high $Q$ in this cavity is to consider that by tuning of the cavity length we alter the phase-matching condition of the two-dimensional Fabry-Perot resonator. Motivated by this reasoning, we investigated alternative methods of tuning the cavity length without introducing any dislocations. One possibility is shown in Fig. 1. Two holes along the $x$ axis, closest to the defect hole, are cut in half, and the halves facing the defect are turned into ellipses while the other two halves are kept the same (half-circles). The equation of the ellipse that we use is $x^{2} /(r-\Delta / 2)^{2}+y^{2} / r^{2}=1$, and therefore the length of the cavity can be expressed as $w=a(2-2 r / a+\Delta / a)$. We show in Fig. 2(a) that, by changing the ellipticity of these ellipses and thereby tuning the cavity length, the $y$ dipole mode can reach $Q \approx 21,000$ when $\Delta / a=40 \%$. This result is obtained with a three-dimensional finite-difference time-domain (FDTD) calculation, with discretization $a=20$ computational points per lattice period. The
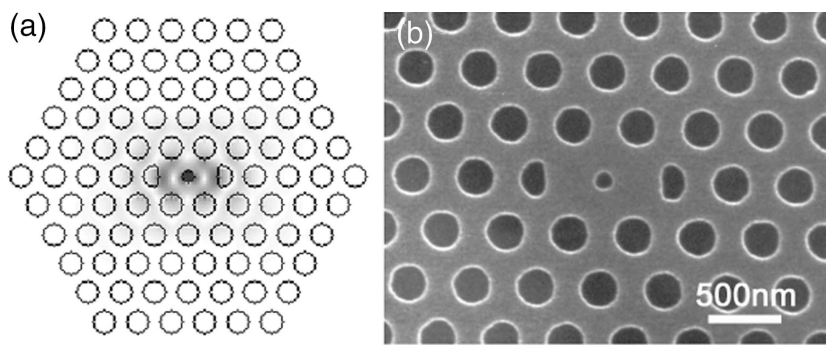

Fig. 1. (a) Cavity design and electric field distribution of the high- $Q$-factor $y$ dipole mode supported in the cavity. (b) Scanning electron microscopy picture of the fabricated cavity. 

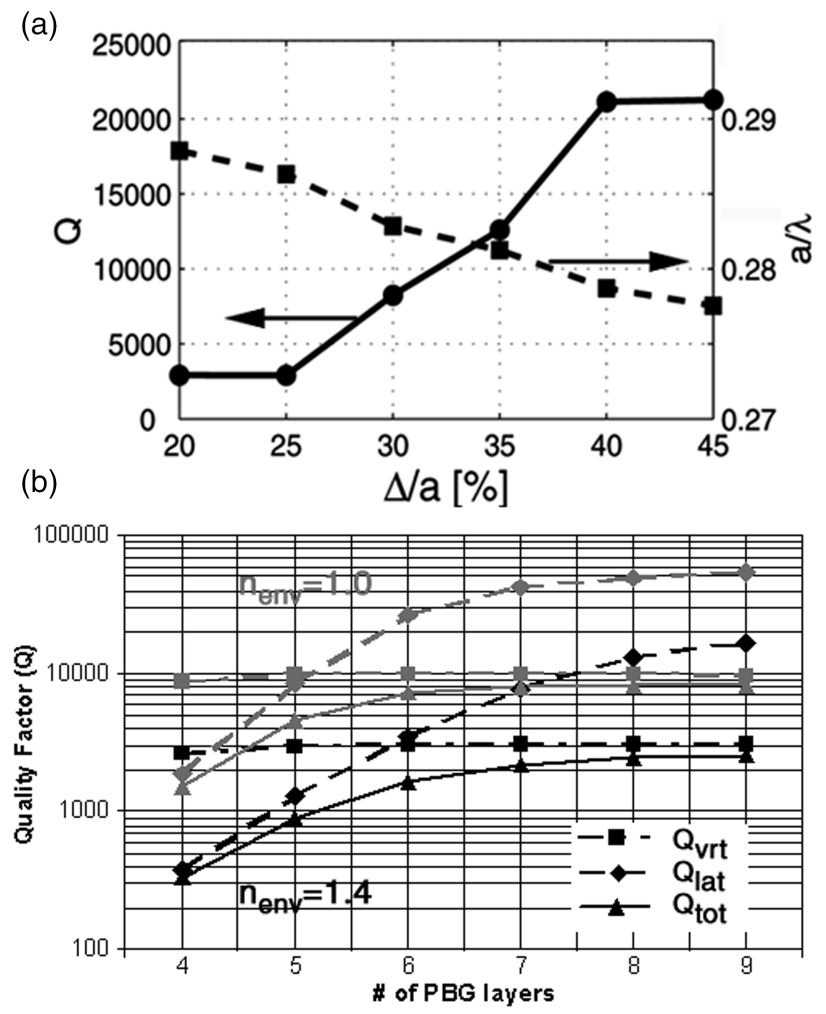

Fig. 2. (a) Dependence of the vertical $Q$ factor on elipticity of two modified holes $(\Delta / a)$. (b) Dependence of the vertical $\left(Q_{\text {vrt }}\right)$, lateral $\left(Q_{\text {lat }}\right)$, and total $\left(Q_{\text {tot }}\right)$, quality factors on the number of photonic crystal layers surrounding the cavity. $1 / Q_{\text {tot }}=1 / Q_{\text {vrt }}+1 / Q_{\text {lat }}$. Cavity with $\Delta / a=40 \%$, immersed in air (gray) and liquid with $n=1.4$ (black) is analyzed.

$Q$ factor shown is the vertical $Q$ factor, since we assume that the lateral $Q$ factor can be increased by addition of more photonic crystal layers. ${ }^{10}$ The $Q$ factor is very sensitive to the size of the central defect hole and drops to $Q_{\mathrm{vrt}} \approx 5500$ and $Q_{\mathrm{vrt}} \approx 8500$ when $r_{\text {def }}=0.1$ and $r_{\text {def }}=0.2$, respectively. However, these lower factors can subsequently be improved as we reoptimize the ellipse shapes $(\Delta / a)$. Larger defect holes, for example, require different cavity lengths (phase shifts) for maintaining high $Q$ modes, as was also shown in previous cavities based on FEDs. ${ }^{11}$

In Fig. 2(b) we show the lateral $\left(Q_{\text {lat }}\right)$, vertical $\left(Q_{\mathrm{vrt}}\right)$, and total $\left(Q_{\text {tot }}\right)$ quality factors for different numbers of photonic crystal layers surrounding the cavity. This time we used discretization $a=15$ computational points. This coarse discretization results in larger effective PPC holes and reduced $Q_{\text {vrt }} \approx 10,000$.

In our cavities and lasers the optical field is the strongest in the air region of the central defect hole (Fig. 1). This is fundamentally different from more conventional photonic crystal laser designs ${ }^{3-5,6,12}$ that localize light in dielectric material. Because of the strong overlap between light and the environment in our cavities, they are natural candidates for use in the investigation of interaction between light and matter introduced in the defect hole. ${ }^{9}$ Therefore, it is of interest to investigate the quality factors of our cavities when they are immersed in environments with a refractive index $\left(n_{\text {env }}\right)$ higher than 1. In Fig. 2(b) we show the results of three-dimensional finitedifference time-domain calculations for $\left(n_{\mathrm{env}}=1.4\right)$. It can be seen that $Q$ factors as high as $Q \approx 3150$ can be achieved in this case. This quality factor is three times higher than $Q$ predicted from cavities by use of FEDs. ${ }^{9}$ However, because of the reduced index contrast between the semiconductor membrane and the environment, more photonic crystal layers are needed to achieve sufficient $Q_{\text {lat }}$. We were even able to achieve $Q_{\text {vrt }} \approx 1800$ when $n_{\mathrm{env}}=1.6$. This reasonably high $Q$ factor suggests that it will be possible to achieve room-temperature lasing when the cavity is surrounded by liquid crystals to tune the emission wavelength efficiently.

Another high- $Q$ cavity design is shown in Fig. 3(a). This time, the four holes closest to the defect (indicated by the arrows) are modified, and the bottoms of the holes above the defect and the tops of the holes below the defect are turned into ellipses. For comparison, in Fig. 3(b) we show our previous high- $Q$ cavity design based on FEDs. The cavity lengths $(w)$ in the two cavities shown in Fig. 3 can be easily compared, and maximum $Q$ in each cavity is reached for similar $w$. However, the $x$ dipole mode can reach $Q \approx 22,600$ in new cavity design, which is a significant improvement over the value $Q \approx 6000$ available from cavity designs based on FED. ${ }^{11}$

Our structures are fabricated in InGaAsP quantumwell material. Optical gain is provided by four 9-nm-thick, compressively strained quantum wells, designed to operate at $\lambda=1.55 \mu \mathrm{m}$. This active material is placed in the center of a $330-n m$-thick InGaAsP slab with a $1-\mu \mathrm{m}$-thick sacrificial InP layer underneath it. An etch mask consists of $170 \mathrm{~nm}$ of $\mathrm{SiO}_{2}$ and $200 \mathrm{~nm}$ of poly(methyl methacrylate) (PMMA) electron-beam resist. Electron-beam lithography is performed using a Leica EBPG 5000+ electron-beam writer, and the structures are developed in a 3:1 solution of isopropyl alcohol and methyl isobutyl ketone. Reactive ion etching (RIE) with $\mathrm{CHF}_{3}$ is used to transfer patterns from PMMA into $\mathrm{SiO}_{2}$, and inductively coupled plasma (ICP) RIE is used to transfer patterns from $\mathrm{SiO}_{2}$ into InGaAsP. We use a mixture of hydrogen iodide, hydrogen, and argon to perform this etch, ${ }^{13}$ with the ratio $\mathrm{HI}: \mathrm{H}_{2}: \mathrm{Ar}=30: 10: 3$ SCCM (SCCM denotes cubic centimeters per minute at STP). This gas chemistry provides smooth and straight sidewalls in quaternary material and does not require heating of the sample, as is typically
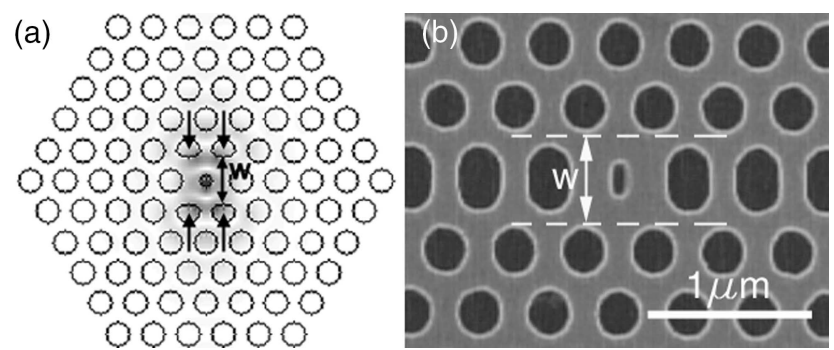

Fig. 3. (a) Alternative cavity design that supports high- $Q$ $x$ dipole modes. (b) Scanning electron microscope picture of the cavity based on FEDs. $w$ is the cavity length. 
(c)

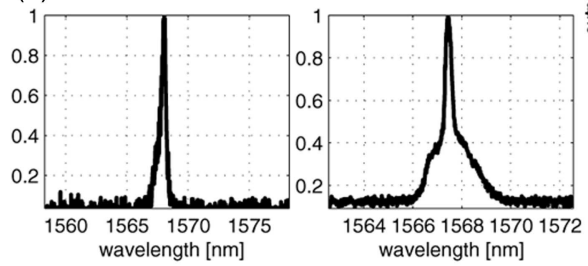

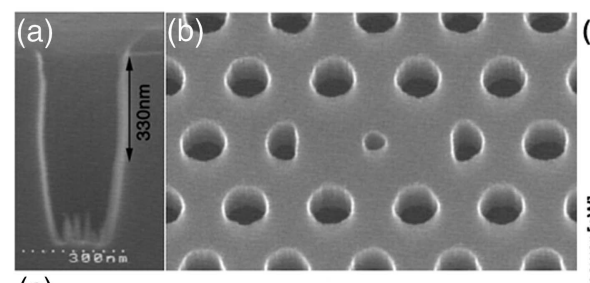

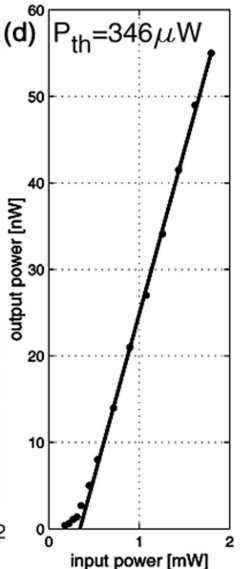

Fig. 4. (a) Cross section of one hole after ICP RIE. (b) $20^{\circ}$ view of the final undercut structure. (c) Spectrum of the laser taken at two different pumping levels. (d) Input power $(\mathrm{L}-\mathrm{L})$ curve.

necessary in chlorine-based chemistries. ${ }^{3-5}$ The chamber pressure is $P=10 \mathrm{~m}$ Torr, and the ICP and the rf power are 850 and $100 \mathrm{~W}$, respectively. The dc bias was $320 \mathrm{~V}$. The etch rate of InGaAsP/InP is $\approx 500 \mathrm{~nm} / \mathrm{min}$, and the selectivity of the semiconductor etch rate over the $\mathrm{SiO}_{2}$ mask erosion rate is better than 8:1. The remaining mask is removed with hydrofluoric acid, and the InGaAsP membrane is subsequently released from the substrate by wet etching in a $4: 1 \mathrm{HCl}$ :water solution at $4^{\circ} \mathrm{C}$. The final structure is a free-standing membrane supported on one side. In Fig. 4(a) we show the cross section of the sample after ICP RIE, and in Fig. 4(b) we show the final undercut structure viewed at a $20^{\circ}$ angle. It can be seen that straight walls are achieved at the position of the InGaAsP slab that contains quantum wells, whereas some overcut is observed in the InP sacrificial layer. The periodicity of the structure characterized here was $a=449 \mathrm{~nm}$, with hole radius $r=129 \mathrm{~nm}$ and defect hole radius $r_{\text {def }}=65 \mathrm{~nm}$. This periodicity results in geometry $d / a \approx 0.73$, $r / a \approx 0.29$, and $r_{\text {def }} / a \approx 0.14$, similar to the results that we characterized numerically.

The cavities are optically pumped with 15-ns-long pulses (with a periodicity of $1.5 \mu \mathrm{s}$ ) from a semiconductor laser diode $\left(\lambda_{\text {pump }}=830 \mathrm{~nm}\right)$. The pump beam is focused through a $100 \times$ objective lens onto the sample surface to obtain a spot size of $\sim 3 \mu \mathrm{m}$. The emission from the cavities is collected through the same lens, and the spectrum of the emitted light signal is detected with an optical spectrum analyzer and an infrared camera. Room-temperature lasing is observed from a number of structures. The spectrum of one laser, taken at two different pumping levels, is shown in Fig. 4(c). The broad background observed when the laser is pumped at higher powers might be attributed to heating of the sample as a result of poor heat sinking of our laser membrane. The dependence of output power on the input power ( $\mathrm{L}-\mathrm{L}$ curve), shown in Fig. 4(d), demonstrates a characteristic lasing threshold of $P_{\mathrm{th}}=346 \mu \mathrm{W}$.

In conclusion, we have proposed, designed, fabricated, and characterized novel planar photonic crystal cavity designs that support dipole modes with high quality factors. Our cavity maintains the symmetry of the triangular lattice and therefore permits easy integration with other photonic crystal devices within the same photonic crystal slab. We have developed fabrication procedures (based on a $\mathrm{HI} / \mathrm{H}_{2} / \mathrm{Ar}$ mixture) that enable us to control precisely the cavity geometries in the InGaAsP material system, with straight and smooth sidewalls. Finally, we have observed room-temperature lasing action from a localized defect mode of the cavity when structures were optically pumped at room temperature, and we predict that these cavities will work well in high-refractive-index ambient environments.

The authors acknowledge interaction with Will Green and Tomo Yoshie from Caltech and Sam Keo from the Jet Propulsion Laboratory. Generous support by the U.S. Air Force Office of Scientific Research (G. Pomrenke) and the Defense Advanced Research Projects Agency (J. Shah) is also acknowledged. M. Lončar's e-mail address is loncar@deas.harvard.edu.

*Present address, Division of Engineering and Applied Sciences, Harvard University, 9 Oxford Street, Cambridge, Massachusetts 02138.

\section{References}

1. J. Vučković, M. Lončar, H. Mabuchi, and A. Scherer, Phys. Rev. E 65, 016608 (2002).

2. J. Vučković and Y. Yamamoto, Appl. Phys. Lett. 82, 2374 (2003).

3. H. Ryu, S. Kim, H. Park, J. Hwang, Y. Lee, and J. Kim, Appl. Phys. Lett. 80, 3883 (2002).

4. H. Park, J. Hwang, J. Huh, H, Ryu, Y. Lee, and J. Kim, Appl. Phys. Lett. 79, 3032 (2001).

5. K. Srinivasan, P. E. Barclay, O. Painter, J. Chen, A. Y. Cho, and C. Gmachl, Appl. Phys. Lett. 83, 1915 (2003).

6. T. Yoshie, O. Shchekin, H. Chen, D. Deppe, and A. Scherer, Electron. Lett. 38, 967 (2002).

7. T. Yoshie, J. Vučković, A. Scherer, H. Chen, and D. Deppe, Appl. Phys. Lett. 79, 4289 (2001).

8. M. Lončar, T. Yoshie, A. Scherer, P. Gogna, and Y. Qiu, Appl. Phys. Lett. 81, 2680 (2002).

9. M. Lončar, A. Scherer, and Y. Qiu, Appl. Phys. Lett. 82, 648 (2003).

10. O. Painter, J. Vučković, and A. Scherer, J. Opt. Soc. Am. B 16, 275 (1999).

11. M. Lončar, T. Yoshie, K. Okamoto, Y. Qiu, J. Vučković, and A. Scherer, "Planar photonic crystal nanolasers: (I) porous cavity lasers," IEICE Trans. E (to be published).

12. O. Painter, R. Lee, A. Scherer, A. Yariv, J. O'Brien, P. Dapkus, and I. Kim, Science 284, 1819 (1999).

13. S. J. Pearton, U. K. Chakrabarti, A. Katz, F. Ren, and T. R. Fullowan, Appl. Phys. Lett. 60, 838 (1992). 